\title{
Instructions, strategies, and pattern uncertainty in a visual discrimination task ${ }^{1}$
}

\author{
DAVID E. CLEMENT ${ }^{2}$ AND CARL F. R. WEIMAN ${ }^{3}$ \\ UNIVERSITY OF SOUTH FLORIDA
}

\begin{abstract}
A card-sorting task involved discrimination of patterns from the same equivalence set, patterns from different sets of the same size, and patterns from different sets of different sizes. Instructions, experimental conditions, and the number of different patterns to be sorted into two trays were varied in an attempt to change Ss' strategies from whole-pattern processing to single-element processing. Instructions alone were ineffectual in preventing whole-pattern processing, and physical masks over most of the criterion stimuli (patterns used as guides in sorting) were only somewhat effective. Differences among tasks that were attributable to size of equivalence set were eliminated only by arranging the stimuli so that a whole-pattern strategy required a great deal more information processing, or so that such a strategy could not lead to the required discrimination. The results were interpreted as confirming the influence of the properties of sets of stimuli, as well as the strength of the tendency of human $S s$ to process and categorize patterns in their entirety.
\end{abstract}

Human visual perception involves selection and categorization of elements and relations in the available stimulus array. Shepard (1966) suggests that searching, grouping, and ordering are continual active functions of the human observer, and Sternberg (1967) has described the encoding of visual stimuli as abstracted representations of their physical properties. These abstracted representations, labeled schema by Evans (1967), are leamed without specific instructions or specific external reinforcement (Edmonds, Mueller, \& Evans, 1966), and thus seem to be the result of very strong processing strategies, at least for adult Ss. Garner (1966) has argued that factors known in perception are properties of sets of stimuli, and encoding processes are operating on such sets, rather than on the single stimulus configuration presented during a single experimental trial. Following Garner's analysis (1962), pattern uncertainty or size of equivalence set has been shown to predict performance in various tasks involving encoding of visual stimuli (e.g., Clement, 1964; Royer, 1966; Handel \&
Garner, 1966; Thurmond \& Alluisi, 1967; Clement, 1967).

Clement and Varnadoe (1967) demonstrated that difficulty of discrimination of patterns such as those in Fig. 1 is predictable from the pattern uncertainty associated with each pattern to be discriminated. The pattern uncertainty was defined by the number of patterns considered equivalent to the pattern under consideration, which is the same as the number of different patterns that can be formed by $90-\mathrm{deg}$ rotations, reflection from left to right, or combinations of these operations. The size of such equivalence sets for the patterns in Fig. 1 can be one (e.g., Pattern 11), four (e.g., Pattern 41), or eight (e.g., Pattern 81). This objective way of determining equivalence sets has been shown to correlate significantly with the actual categorization of such patterns by human Ss (Garner \& Clement, 1963). In the Clement and Varnadoe study, each S sorted eight decks of 50 cards, containing 25 cards with each of two different patterns. The patterns to be discriminated could be from within the same equivalence set (e.g., Pattern 43 and a reflection of that pattern), from different equivalence sets of the same size (e.g., Pattern 41 and Pattern 42), or from different equivalence sets of different sizes (e.g., Pattern 11 and Pattern 81). In addition, the patterns in each deck were in a "fixed" orientation for one group of Ss (i.e., each pattern in the deck was oriented in the same manner as one of the two criterion patterns mounted above the trays used for sorting), and in a "random" orientation for a second group of Ss (i.e., patterns in the deck could be turned $0,90,180$, or $270 \mathrm{deg}$ from the criterion patterns). The results indicated that sorting time increased with increasing size of equivalence set for patterns from different sets of the same size, and sorting time was greater for patterns from within the same set than for patterns from different sets, in accordance with the experimental hypotheses. A post hoc analysis of sorting times for patterns from sets of different sizes indicated that Ss were responding to an average pattern uncertainty rather than to the difference in uncertainty associated with the two patterns in the deck. An interaction between orientation of patterns in the deck (fixed or random) and the discrimination tasks took the form of greater differences (a steeper slope of sorting time plotted against task) for the random orientation. Clement and Varnadoe interpreted this as a natural concomitant of the need to match orientation between patterns in the deck and criterion patterns, but suggested that it could be due in part to the use of a different kind of processing strategy for patterns in the fixed orientation. Unlike the random orientation, Ss with the fixed orientation of patterns did not have to process the entire pattern, but rather could focus their attention on any of the nine possible dot locations that differed (dot or no dot) between the two patterns. Exclusive use of this strategy by some Ss, or occasional use by all Ss, would have attenuated differences between the tasks, leading to the obtained lower slope for fixed orientation. Ordinal consistency of the predicted relations within Ss argued against extensive use of a strategy other than whole-pattern processing, but not against occasional or inefficient use of an alternative strategy.

This study was designed to investigate the possible use of different processing strategies, manipulating them by means of the instructions given to the Ss. Different conditions were designed to give increasing amounts of instructional bias toward processing individual elements in the patterns. The hypothesis was that increasing element-processing bias would decrease the differences in sorting times among the discrimination tasks used in the Clement and Vamadoe (1967) study.

\section{Subjects}

\section{METHOD}

Sixty-eight students (36 male, 32 female) from the introductory course in psychology were used as Ss.

\section{Stimuli}

The patterns used as stimuli were taken from Garner and Clement (1963), and are shown in Fig. 1. The patterns were printed in black, centered on $3 \times 3$ in. white cards. Each dot was $1 / 8$ in. in diam, centered in a 5/16-in.-square cell (cells were arranged in a 3 by 3 square matrix, with no boundary lines actually present on the stimulus cards). 


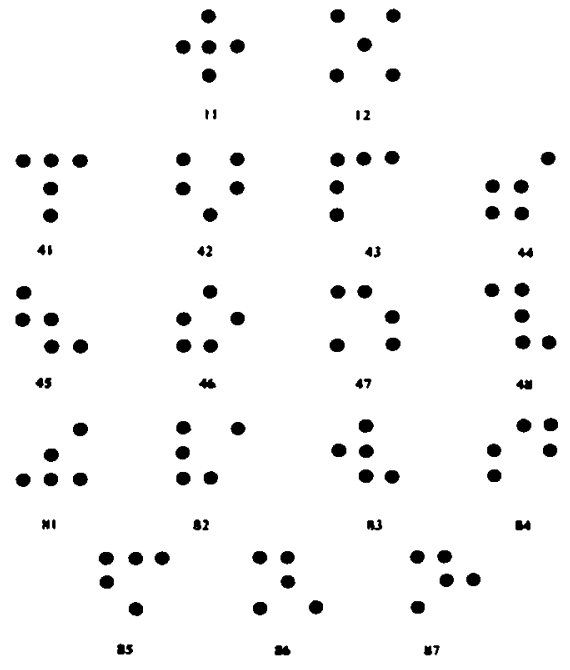

Fig. 1. Dot patterns used as stimuh.

The first number in the pattern designation in Fig. 1 refers to the size of the equivalence set to which the pattern belongs; the second number is an identification number for that set. The letters $x$ and $y$ in Table 1 refer to different patterns from different sets; the letters a and $b$ refer to different patterns from within the same set (e.g., $4 x-4 y$ could refer to a deck with Patterns 41 and $42,4 a-4 b$ to a deck with Pattern 43 and the reflection of that pattern).

\section{General Experimental Task}

The Ss were run individually. Each $S$ was told, by tape-recorded instructions, that he would be required to sort the cards in each of eight shuffled decks of cards into two trays, one deck at a time. Mounted on a vertical surface at the rear of each tray was a card bearing one of the two stimulus patterns that were included in each deck, and the $S$ was instructed to put each card into the tray in front of the pattern that it matched (the criterion stimulus). The $S$ was told to proceed as fast as possible while avoiding errors. Two warm-up trials were run with a deck of black cards and white cards prior to the eight experimental trials. In each trial, the $E$ placed a covered deck of cards in front of the $S$, then uncovered the criterion stimuli. The order to begin was given, and the duration of the trial was timed. Sorting errors were recorded after each $S$ had finished. Order of tasks, location of criterion stimuli (left or right), and specific patterns used in each deck were counterbalanced across Ss. The Ss were given no information about their performance until completion of the experiment.

Condition 1. Twenty Ss (15 male, S female) were run in this condition. Each of the eight decks of cards contained 50 cards (25 each of two different patterns). The eight discrimination tasks were those listed in Table 1 , as in the Clement and Varnadoe (1967) study. The orientation of the cards in the deck was fixed, matching the orientation of the criterion cards. The instructions were the same as in the Clement and Varnadoe study, with the addition of instructions to "look at the entire pattern, not just a few of the dots, for many times the choice is much easier to make if you look at the whole pattern." Presumably, this was a replication of the fixed orientation part of the Clement and Varnadoe study, with instructional modifications to minimize single-element processing.

Condition 2. Twenty different Ss (10 male, 10 female) were run exactly as in Condition 1, except that the added instructions were to "try to find a single dot which is different in the two patterns. Then, when you sort each card, look for the key dot and do not waste time looking at the rest of the pattern." This instruction was intended to focus processing on a single dot location. If effective, differences in sorting times between discrimination tasks should have been reduced to chance values.

Condition 3. After examining the results from the first two conditions (discussed later), three more conditions were designed, in an effort to force single-element processing. This condition was run with 10 different Ss ( 2 male, 8 female). A masking card was placed over each of the two criterion cards, covering all but one corner (one cell that might contain a dot) of each card. The chosen corner was one that contained a dot for one of the patterns and no dot for the other pattern. Actually, the patterns used as criterion patterns were never changed, since only one corner of each was visible during the trials. Location of the comer was randomized across Ss and trials. Instructions were the same as in Condition 2, except that the location of a key dot was thus specified in advance for each $S$ in each task. Forty-eight cards were in each deck (24 each of two different patterns in a fixed orientation).

Condition 4. Ten different Ss (5 male and 5 female) were run with the same instructions and same physical situation as in Condition 3. The eight decks of cards, however, were formed in a different manner. Each deck contained 48 cards, six each of eight different patterns from sets of the same size. Each pattern was in a fixed orientation throughout the deck, and the patterns were chosen so that four patterns had a dot in the key location and four did not. Four decks for each $S$ contained patterns from sets of Size 4, while the other four decks contained patterns from sets of Size 8 . If the $S$ were to process the whole pattern, he would have to learn which four patterns went into one tray and which four patterns went into the other-a much more difficult task than in the previous conditions. This should have made it more likely that the Ss would follow the instructions, and concentrate on the key dot location. Thus, differences between decks of patterns from sets of Size 4 and decks of patterns from sets of Size 8 should have been minimal.

Condition 5. Ten different Ss ( 4 male, 6 female) were run with the same instructions and physical situation as in Conditions 3 and 4 . The eight decks consisted of four with patterns from sets of Size 4 and four with patterns from sets of Size 8. Each deck, however, contained only patterns from a single equivalence set (actually rotations of a single pattern), and the cards were oriented randomly in each deck, with the single restriction that 24 cards had a dot in the key location, while 24 did not. Thus, all cards in a given deck contained patterns that could be superimposed by rotation, and the $S$ could not discriminate the correct sorting response on the basis of pattern, but was forced to follow the single-element instructions.

\section{RESULTS}

The results of the five experimental conditions are summarized in Table 1 . The dependent variable, sorting time, was positively skewed, so median sorting time was considered the appropriate measure of central tendency for each $S$. The mean value of median sorting times did not vary significantly across experimental conditions, but the variability across Ss within conditions made comparisons of actual times confusing. Therefore, the times were transformed for each $S$ into deviations from median sorting time, henceforth called "deviation times." These deviation times reflect individual differences in dispersion but not in central tendency across Ss.

Very few errors were made by Ss except in the $4 a-4 b$ and $8 a-8 b$ tasks, so error data were not subjected to analysis.

\section{Condition 1}

For the five discrimination tasks involving patterns from sets of the same size (Tasks ae in Table 1), the Ss performed in about the same way as those in the fixed orientation condition of the Clement and Varnadoe (1967) study, though the deviation times were somewhat 
Table 1

Mean Deviations from Median Sorting Times (in Sec)

\begin{tabular}{|c|c|c|c|c|c|}
\hline \multirow{2}{*}{$\begin{array}{l}\text { Discrimination } \\
\text { Task }\end{array}$} & \multirow[b]{2}{*}{1} & \multicolumn{3}{|c|}{ Experimental Conditions } & \multirow[b]{2}{*}{$5^{*}$} \\
\hline & & 2 & 3 & $4 *$ & \\
\hline $\begin{array}{l}\text { (a) } 1 x-1 y \\
\text { (b) } 4 x-4 y \\
\text { (c) } 8 x-8 y \\
\text { (d) } 4 a-4 b \\
\text { (e) } 8 a-8 b\end{array}$ & $\begin{array}{r}-3.0 \\
-.8 \\
4.0 \\
4.1 \\
9.3\end{array}$ & $\begin{array}{r}-1.7 \\
-.2 \\
.2 \\
4.5 \\
6.5\end{array}$ & $\begin{array}{r}-.7 \\
-.2 \\
.8 \\
2.5 \\
3.1\end{array}$ & $\begin{array}{r}.9 \\
1.2 \\
-- \\
--\end{array}$ & $\begin{array}{r}-- \\
-- \\
-- \\
.6 \\
.4\end{array}$ \\
\hline $\begin{array}{l}\text { (f) } 1 x-4 y \\
\text { (g) } 1 x-8 y \\
\text { (h) } 4 x-8 y\end{array}$ & $\begin{array}{r}-2.7 \\
-2.0 \\
.6\end{array}$ & $\begin{array}{r}-2.0 \\
-1.8 \\
1.8\end{array}$ & $\begin{array}{r}-2.4 \\
-1.3 \\
.9\end{array}$ & $\begin{array}{l}-- \\
-- \\
--\end{array}$ & $\begin{array}{l}-- \\
-- \\
--\end{array}$ \\
\hline $\begin{array}{l}\text { Mean of median } \\
\text { sorting times } \\
\text { (No. Ss) }\end{array}$ & $\begin{array}{l}36.6 \\
(20)\end{array}$ & $\begin{array}{l}33.6 \\
(20)\end{array}$ & $\begin{array}{l}33.8 \\
(8)\end{array}$ & $\begin{array}{l}41.3 \\
(10)\end{array}$ & $\begin{array}{l}36.1 \\
(10)\end{array}$ \\
\hline
\end{tabular}

- The tasks in these conditions used more than two patterns, as discussed in the text.

less for the $4 a-4 b$ and $8 a-8 b$ discrimination tasks. The ordering of the mean deviation times was the same in the two studies (Spearman rank-order correlation or rho $=1.0, p<.01$ ), and the averaged tho for all $20 \mathrm{Ss}$ was .73 , also significant at the .01 level and somewhat higher than in the previous study. A repeated measures analysis of variance of the deviation times showed the differences among tasks to be significant $[F(4,72)=12.57, p<.01]$, while the effects of sex of $S$ and the interaction between tasks and sex of $S$ failed to reach significance at the .05 level.

For the three discrimination tasks involving patterns from sets of different sizes (Tasks $f-h$ in Table 1), analogous results were obtained. The ordering of mean deviation times was the same as in the Clement and Varnadoe (1967) study, with an averaged tho for all 20 Ss of .42 , indicating more inter-S variability than for the other five discrimination tasks. An analysis of variance of deviation times yielded only a significant task effect $[F(2,36)=4.58, p<.05]$

\section{Condition 2}

Differences in deviation times were less under this condition for Tasks a-e, although the ordering of mean deviation times remained identical to that previous $\mathbf{N}$ obtained (rho $=1.0, p<.01$ ), and the averaged tho for all 20 Ss was .70 (signifiçant at the .01 level). A repeated measures analysis of variance of deviation times yielded only a significant task effect $[F(4,72)=12.15, p<.01]$. Many Ss indicated difficulty in ignoring the whole pattern, although all claimed to have learned to do so by the end of the first task.

For Tasks $f-h$, analogous results were obtained. The ordering of mean deviation times was identical to that obtained before with an averaged tho for all 20 Ss of .48 , and an analysis of variance yielded only a significant task effect $[F(2,36)=9.03$, $\mathrm{p}<.01\}$.

\section{Condition 3}

Differences in deviation times for Tasks a were attenuated even more in this condition, although the ordering of mean deviation times remained identical to previous orderings (rho $=1.0, \mathrm{p}<.01$ ). The criterion mask seemed to affect $\mathrm{Ss}$ differentially, however, for the averaged rho for eight Ss was only .42 , significant at the .05 level but below that for prior conditions. In addition, a repeated measures analysis of variance failed to yield any factors significant at the .05 level. The difficulties encountered by the $S s$ in attending to the single dot location were particularly salient for two Ss (both female) who claimed that there were no differences among patterns in the deck on Task $e$, even after the $E$ had repeated the instructions concerning identification of the single dot that was present or absent in one key location. These two Ss placed all the cards for that deck into one tray without attempting to sort them (all data for these Ss were eliminated before analysis). The other Ss made statements about the difficulty of ignoring the whole patterns, but, as in Condition 2, ciaimed to have successfully overcome this.

For Tasks $f-h$, similar results were obtained. The ordering of mean deviation times was identical to that from prior conditions, with an averaged tho of .42 for the eight Ss. An analysis of variance yielded no significant effects.

\section{Condition 4}

There was no significant difference in mean deviation times between decks containing only patterns from different sets of Size 4 and decks containing only patterns from different sets of Size 8 . The data were condensed into a fourfold frequency matrix, with set size as one factor and direction of deviation time from the median as the other factor. The phi coefficient for the resulting matrix was .02 , trivially different from zero. The familiar $S$ comments concerning difficulty of ignoring the whole pattem were again received.
Condition 5

There was no significant difference in mean deviation times between decks containing rotations of a pattern from a set of Size 4 and decks containing rotations of a pattern from a set of Size 8. A matrix obtained as in Condition 4 yielded a phi c efficient of .15 , again not significantly different from zero. The Ss again voiced the difficulty of ignoring the whole pattern initially, although they seemed less distracted than those in Condition 4, where more than one pattern was used.

\section{DISCUSSION}

The results confirm the powerfulness of the tendency for human Ss to process entire visual patterns and to utilize the uncertainty of the equivalence sets to which the patterns belong. Instructions alone were ineffectual in preventing this whole-pattern processing, as demonstrated by the similarity in results for Conditions 1 and 2. Sexton and McLaughlin (1967) found that instructions had less effect on performance for Ss who encoded certain multidimensional stimuli with a syntactic phrase (a kind of pattern encoding) than for Ss who encoded the same stimuli by separate stimulus dimensions (a kind of elemental encoding). This finding is completely consonant with the results of the present study, if it is assumed that all Ss in Condition 2 encoded by pattern rather than by element.

Emphasizing a key element by use of a mask on the criterion (Condition 3) was somewhat effective in reducing whole-pattern processing, though it did not prevent significant performance differences predictable on the basis of size of equivalence set. The effect of the mask operated differentially across Ss, as indicated by the decrease in average tho from the values in previous conditions. Differences in deviation times attributable to the effects of differential pattern uncertainty were eliminated only by arranging the stimuli so that a whole-pattern processing strategy required a great deal more information processing (Condition 4), or so that such a strategy could not lead to the required discrimination (Condition 5).

The consistent ordering of deviation times for Tasks $f$ h provides some support for the post hoc suggestion by Clement and Varnadoe (1967) that both patterns in a given task are processed, with performance being related to same kind of averaged uncertainty for patterns from equivalence sets of different sizes.

Egeth (1967) summarized data demonstrating that $S s$ are capable of effective gating of irrelevant information consisting of additional stimulus elements, 
but cautioned that such filtering might be achieved easily by $S s$ if the irrelevant attributes did not produce competing implicit responses. The data from the present study indicate that whole-pattern processing is a very strong implicit response, and filtering of part of the pattern consequently is quite difficult. It is interesting to note that most Ss claimed difficulty in ignoring the other dots in the pattern in Conditions 2-5, but also claimed that they were able to accomplish this after the first task. For Conditions 2 and 3, this claim is not supported by the data, suggesting that the Ss were processing the whole pattern even when they were not intending to do so or even aware of the fact that they were doing so. This emphasizes that in this type of task, the $E$ must be careful to evaluate the experimental situation as it appears to the $S$, but should be reluctant to accept verbal statements from the $S$ as accurate descriptions of the perception involved.

The strength of whole-pattern processing tendencies found in this study may not be generalizable to other species, or even to young human Ss. Polidora (1966) found that rhesus monkeys (Macaca mulatta) consistently learned a pattern discrimination by use of unique elements rather than by use of the entire patterns. Brown and Goldstein (1967) obtained data indicating that 5- and 6-year-old human Ss encode stimuli on the basis of specific pattern attributes as well as information content. Whether this is similar to the use of specific pattern attributes by adults, which is suggested by Evans (1967; i.e., a schema is encoded first, then variations from it are encoded), or represents a developmentally earlier set of strategies has not been determined.

Visual stimuli are encoded in their entirety. Further, they are categorized and encoded in sets. Instructions and logical geometric strategies are not in themselves sufficient to overcome this predisposition.

\section{REFERENCES}

BROWN, D. R., \& GOLDSTEIN, J. A. Stimulus correlates of discrimination behavior of children. Psychonomic Science, 1967, 9, 177-178.

CLEMENT, D. E. Uncertainty and latency of verbal naming responses as correlates of pattern goodness. Journal of Verbal Learning \& Verbal Behavior, 1964, 3, 150-157.

CLEMENT, D. F. Paired-associate leaming as a correlate of pattern goodness. Joumal of Verbal Learning \& Verbal Behavior, 1967, 6, 112-116.

CLEMENT, D. E., \& VARNADOE, K. W. Pattern uncertainty and the discrimination of visual patterns. Perception \& Psychophysics, 1967, 2, 427-431.

EDMONDS, E. M., MUELLER, M. R., \& EVANS, S. H. Effects of knowledge of results on mixed schema discrimination. Psychonomic Science, 1966, 6, 377-378.

EGETH, H. Selective attention. Psychological Bulletin, 1967, 67, 41-57.

EVANS, S. H. A brief statement of schema theory. Psychonomic Science, 1967, 8, 87-88.

GARNER, W. R. Uncertainty and structure as psychological concepts. New York: Wiley, 1962.
GARNFR, W. R. To perceive is to know. American Psychologist, 1966, 21, 11-19.

GARNER, W. R., \& CLEMENT, D. E. Goodness of pattern and pattern uncertainty. Journal of Verbal Learning \& Verbal Behavior, 1963,2 446-452.

HANDEL, S., \& GARNER, w. R. The structure of visual pattern associates and pattern goodness. Perception \& Psychophysics, 1966, 1, 33-38.

POLIDORA, V. J. Stimulus correlates of visual pattern discrimination by monkeys Multidimensional analyses. Perception \& Psychophysics, 1966, 1, 405-414.

ROYER, F. L. Figural goodness and internal structure in perceptual discrimination. Perception \& Psychophysics, 1966, 1, 311-314.

SEXTON, E., \& McLAUGHLIN, J. P. Set and the encoding of visual stimuli. Psychonomic Science, 1967, 9, 219-220.

SHEPARD, R. N. Learning and recall as organization and search. Journal of Verbal Learning \& Verbal Behavior, 1966, 5, 201-204.

STERNBERG, S. Two operations in character recognition: Some evidence from reaction-time measurements. Perception \& Psychophysics, 1967, 2, 45-53.

THURMOND, J. B., \& ALLUISI, E. A. An extension of the information-deductive analysis of form. Psychonomic Science, 1967 7, 157-158.

\section{NOTES}

1. This research was supported in part by a University Research Council grant.

2. Address: Department of Psychology, University of South Florida, Tampa, Florida 33620 .

3. Now at The Ohio State University.

(Accepted for publication September 30, 1969.) 